\title{
Computationally Efficient FBP-type Direct Segmentation of Synthetic Aperture Radar Images
}

\author{
H. Cagri Yanik, Zhengmin Li and Birsen Yazıcı \\ Electrical, Computer, and System Engineering Department, Rensselaer Polytechnic Institute, \\ Troy, NY 12180 USA
}

\begin{abstract}
We consider a monostatic synthetic aperture radar system traversing an arbitrary trajectory on a non-flat topography. We present a novel edge detection method applicable directly to SAR received signal. Our method first filters the received data, and then backprojects. The filter is designed to detect the edges of the scene in different directions at each pixel reconstructed. The method is computationally efficient and may be implemented with the computational complexity of the fast-backprojection algorithms. We present numerical experiments to demonstrate the performance of our method.
\end{abstract}

Keywords: Imaging, Segmentation, Synthetic Aperture Radar, Backprojection

\section{INTRODUCTION}

In many applications, synthetic aperture radar (SAR) images are subjected to automated pattern recognition algorithms that require segmentation of the reconstructed images. Segmentation is an important image processing task to recognize objects or edges in a SAR image, such as houses, vehicles etc. Segmentation can be addressed by different methods such as clustering and boundary detection-type algorithms.

In this paper, we introduce a filtered-backprojection (FBP) type image segmentation method applied to SAR received signal. The method detects edges directly from received signal and bypasses the image reconstruction step. We first model the SAR received signal with an arbitrary flight trajectory as a Fourier Integral Operator (FIO). We next present a FBP-type method to recover and enhance the visible edges of the scene to be imaged. The FBP-type segmentation involves a backprojection step that is given by the $L^{2}$-adjoint of the phase of the FIO and a filtering step. We design the filter so that the resulting point spread function of the FBP operator is a differential operator that reconstructs the derivative (or anti-derivative) of the image in desired directions for each pixel reconstructed.

While we focus primarily on the segmentation of SAR images, the method is applicable to other type of imaging applications where the projection data are modeled as FIOs, such as synthetic aperture sonar and geophysical imaging.

This paper is organized as follows: In Section 2, the SAR forward model and generalized FBP method are described. In Section 3, a brief discussion on image segmentation via edge detection is given. In Section 4, a new filter that reconstructs the edge map of the scene is derived. In Section 5, numerical results are provided to demonstrate the performance of this method. Finally, we conclude our discussion in Section 6.

Further author information: (Send correspondence to Birsen Yazıcı)

Birsen Yazıcı: E-mail: yazici@ecse.rpi.edu

H. Cagri Yanik: E-mail: yanikh@rpi.edu

Zhengmin Li: E-mail: liz7@rpi.edu 


\section{FORWARD MODELING AND GENERALIZED FILTERED-BACKPROJECTION}

Unless otherwise stated, the bold Roman, bold italic, and Roman small letters will denote points in $\mathbb{R}^{3}, \mathbb{R}^{2}$ and $\mathbb{R}$, respectively, i.e. $\mathbf{x}=\left(\boldsymbol{x}, x_{3}\right) \in \mathbb{R}^{3}$, with $\boldsymbol{x} \in \mathbb{R}^{2}$, and $x_{3} \in \mathbb{R}$.

Let $\gamma(s) \in \mathbb{R}^{3}$ denote the flight trajectory of the antenna, where $s \in \mathbb{R}$ is the slow-time variable, parameterizing the trajectory. We assume that the earth's surface is located at the position $\mathbf{x}=\left(x_{1}, x_{2}, \boldsymbol{\psi}\left(x_{1}, x_{2}\right)\right)$, where $\boldsymbol{\psi}: \mathbb{R}^{2} \rightarrow \mathbb{R}$, is a known smooth function, and scattering takes place in a thin region near the surface. Following monostatic version of ${ }^{1}$ and under the single scattering (Born) approximation, we model the received signal $d(s, t)$ as follows:

$$
d(s, t) \approx \mathcal{F}[T](s, t)=\int \mathrm{e}^{-\mathrm{i} 2 \pi \omega\left(t-R(s, \mathbf{x}) / c_{0}\right)} A(\boldsymbol{x}, \omega, s) T(\boldsymbol{x}) \mathrm{d} \omega \mathrm{d} \boldsymbol{x}
$$

where $\boldsymbol{x}=\left(x_{1}, x_{2}\right), R(s, \mathbf{x})=2|\gamma(s)-\mathbf{x}|$ is the total travel distance, $t$ denotes the fast-time, $c_{0}$ denotes the speed of light in vacuum, $T(\boldsymbol{x})$ denotes the surface reflectivity, and $A$ is a complex amplitude function that depends on antenna beam pattern, the transmitted waveform, geometrical spreading factors, etc. ${ }^{2}$

We assume that for some $m_{A}, A$ satisfies the symbol estimate

$$
\sup _{(s, \boldsymbol{x}) \in K}\left|\partial_{\omega}^{\alpha} \partial_{s}^{\beta} \partial_{x_{1}}^{\rho_{1}} \partial_{x_{2}}^{\rho_{2}} A(\boldsymbol{x}, \omega, s)\right| \leq C_{0}\left(1+\omega^{2}\right)^{\left(m_{A}-|\alpha|\right) / 2}
$$

where $K$ is any compact subset of $\mathbb{R} \times \mathbb{R}^{2}$, and the constant $C_{0}$ depends on $K, \alpha, \beta, \rho_{1}$, and $\rho_{2}$. This assumption is needed to make various stationary phase calculations hold; and it also makes the forward operator $\mathcal{F}$ a Fourier Integral Operator (FIO). ${ }^{3-5}$

The reconstruction of the target reflectivity, $T(\boldsymbol{x})$ requires inversion of $\mathcal{F}$. Since $\mathcal{F}$ is an FIO, an approximate inverse of $\mathcal{F}$ by another FIO, $\mathcal{K}$ can be formed such that

$$
T(\boldsymbol{z}) \approx \tilde{T}(\boldsymbol{z})=\mathcal{K} \mathcal{F}[T](\boldsymbol{z})
$$

Let $\mathcal{L}=\mathcal{K} \mathcal{F}$. The kernel $L$ of $\mathcal{L}$ is the Point Spread Function (PSF) of the system which relates the target scene $T(\boldsymbol{x})$ to the reconstructed scene $\tilde{T}(\boldsymbol{z})$. For a perfect reconstruction, $L$ must be a Dirac-delta function. Thus, we choose $\mathcal{K}$ so that the kernel of $\mathcal{L}$ becomes as close to Dirac-delta function as possible.

It was shown in ${ }^{1}$ that a FBP-type operator $\mathcal{K}$ can be designed to reconstruct an image of the scene

$$
\tilde{T}(\boldsymbol{z})=\mathcal{K}[d](\boldsymbol{z}):=\int \mathrm{e}^{\mathrm{i} 2 \pi \omega\left(t-R(s, \mathbf{z}) / c_{0}\right)} Q(\boldsymbol{z}, \omega, s) d(s, t) \mathrm{d} \omega \mathrm{d} s \mathrm{~d} t .
$$

Thus, the PSF of the imaging operator $\mathcal{K}$ is

$$
L(\boldsymbol{z}, \boldsymbol{x})=\int \mathrm{e}^{\mathrm{i} 2 \pi \omega \phi(\boldsymbol{x}, \mathbf{z}, s)} Q(\boldsymbol{z}, s, \omega) A(\boldsymbol{x}, s, \omega) \mathrm{d} \omega \mathrm{d} s
$$

where filter $Q$ is chosen such that $L(\boldsymbol{z}, \boldsymbol{x})=\boldsymbol{\delta}(\boldsymbol{z}-\boldsymbol{x})$ and $\phi$ is given by $\phi(\boldsymbol{x}, \boldsymbol{z}, s)=R(s, \mathbf{x})-R(s, \mathbf{z})$. In this paper, we design a new filter $\bar{Q}$ to form the edge map of SAR images. The edge map image can be viewed as an approximation to the magnitude of the gradient of the image, i.e. $|\nabla T(\boldsymbol{z})|$.

\section{SEGMENTATION VIA EDGE DETECTION}

Segmentation is an important image processing task to extract and locate objects (lines, curves, points etc.) from a given image. These objects are distinctive features of the image and they make it easier to describe, characterize and give meaning to the image.

A basic image segmentation process requires assigning a label to every pixel in an image. Pixels with the same label have similar characteristics. There are many ways to perform image segmentation such as level set methods, graph portioning methods, clustering methods, region growing methods and edge detection. In this 
paper, we segment the SAR image via edge detection by applying a differential filter directly to the SAR data at the backprojection step.

Edges can vaguely be defined as points at which the transition in pixel value associated with that point is significantly stronger than the background at that point. It is well known from vector calculus that the gradient vector of a scalar field is a vector field and this vector field points in the direction of the greatest rate of change of the scalar field. Moreover, magnitude of the vector field corresponds to the greatest rate of change. Thus, edge detection is closely related to the gradient of the image.

A simple method to perform edge detection is by applying an operator such as Prewitt, Sobel and then thresholding the image. ${ }^{6}$ In $^{7}$ Canny proposed an edge detection method that contains several steps: First, image is smoothed with a Gaussian to get rid of the noise and then its gradient is calculated. Depending on the magnitude of the gradient and the angle of the gradient, pixels are labeled, image is thresholded and finally the edge map is formed.

For segmentation of SAR images, the methods described above can be applied after forming the SAR image. In this paper, we introduce a FBP-type image segmentation method applied directly to SAR received signal. The method involves derivation of an edge enhancing (or smoothing) filter and the backprojection operator. The new method reconstructs an approximation to the magnitude of the gradient of the scene, $|\nabla T|$. With this new filter, PSF of the system can be viewed as an approximation to the derivative (or anti-derivative) of the Dirac-delta function

$$
L(\boldsymbol{z}, \boldsymbol{x}) \approx \sum_{i} \alpha_{i} \boldsymbol{\delta}^{\left(p_{i}\right)}(\boldsymbol{z}-\boldsymbol{x})
$$

where $\alpha_{i}>0$ and $p_{i} \in \mathbb{R}$. Here, $p_{i}$ stands for the $p_{i}^{t h}$ derivative and $\alpha_{i}$ controls the strength of the edges in the final image.

\section{DIRECT SEGMENTATION OF THE SAR DATA}

In this section we derive a new filter to enhance (or smooth) the edges in the image. This filter is applied prior to the bacprojection step directly to SAR data.

\subsection{Derivation of an Edge Detection Filter}

In this subsection, we derive a new filter $\bar{Q}$ so that the PSF of the imaging system behaves like the derivative of the Dirac-delta function. Thus, what we reconstruct from SAR data will be approximately the edge map of the scene. The filter that results in an approximate Dirac-delta function PSF is given as

$$
Q(\boldsymbol{z}, \omega, s)=\chi_{\Omega_{\boldsymbol{z}}}(\boldsymbol{\xi}(\omega, s)) \frac{\overline{A(\boldsymbol{z}, \boldsymbol{\xi}(\omega, s))}}{|A(\boldsymbol{z}, \boldsymbol{\xi}(\omega, s))|^{2}} \frac{1}{\eta(\boldsymbol{\xi}(\omega, s), \boldsymbol{z})}
$$

where $\eta(\boldsymbol{\xi}(\omega, s), \boldsymbol{z})$ is the Jacobian that comes from change of variables

$$
(\omega, s) \rightarrow \boldsymbol{\xi}=\frac{\omega}{c_{0}} \nabla_{\boldsymbol{z}} R(s, \boldsymbol{z}) .
$$

With this choice of filter, the PSF of the imaging system is given by

$$
L(\boldsymbol{z}, \boldsymbol{x})=\int \mathrm{e}^{\mathrm{i} 2 \pi(\boldsymbol{x}-\boldsymbol{z}) \cdot \boldsymbol{\xi}} \chi_{\Omega_{z}}(\boldsymbol{\xi}) \mathrm{d} \boldsymbol{\xi} .
$$

Here $\chi_{\Omega_{z}}$ is a smooth cut-off function equal to one in the interior of $\Omega_{\boldsymbol{z}}=\left\{\boldsymbol{\xi}(\omega, s, \boldsymbol{z}) \mid A_{T R}(\boldsymbol{z}, s, \omega) \neq 0\right\}$ and zero in the exterior of $\Omega_{\boldsymbol{z}}$, data collection manifold. 
Here we modify the PSF so that when the resulting filter is applied directly to the SAR received data it reconstructs the edge map of the scene, $T(\boldsymbol{z})$. Recall that the relation between actual scene, $T(\boldsymbol{x})$ and reconstructed scene $T(\boldsymbol{z})$ is given by

$$
\begin{aligned}
\tilde{T}(\boldsymbol{z}) & =\mathcal{L}[T](\boldsymbol{z})=\int L(\boldsymbol{z}, \boldsymbol{x}) T(\boldsymbol{x}) \mathrm{d} \boldsymbol{x} \\
& =\iint_{\Omega_{z}} \mathrm{e}^{\mathrm{i} 2 \pi(\boldsymbol{x}-\boldsymbol{z}) \cdot \boldsymbol{\xi}} \chi_{\Omega_{z}}(\boldsymbol{\xi}) T(\boldsymbol{x}) \mathrm{d} \boldsymbol{\xi} \mathrm{d} \boldsymbol{x} \\
& =\int_{\Omega_{z}} \mathrm{e}^{-\mathrm{i} 2 \pi \boldsymbol{z} \cdot \boldsymbol{\xi}} \chi_{\Omega_{z}}(\boldsymbol{\xi}) \hat{T}(\boldsymbol{\xi}) \mathrm{d} \boldsymbol{\xi}
\end{aligned}
$$

where $\hat{T}(\boldsymbol{\xi})$ stands for the Fourier transform of $T(\boldsymbol{x})$.

To segment the SAR image we wish to reconstruct approximately $|\nabla T(\boldsymbol{z})|$. If we apply the gradient operator to both sides

$$
\begin{aligned}
\nabla_{\boldsymbol{z}} \tilde{T}(\boldsymbol{z}) & =\int_{\Omega_{z}} \nabla_{\boldsymbol{z}} \mathrm{e}^{-\mathrm{i} 2 \pi \boldsymbol{z} \cdot \boldsymbol{\xi}} \chi_{\Omega_{z}}(\boldsymbol{\xi}) \hat{T}(\boldsymbol{\xi}) \mathrm{d} \boldsymbol{\xi}, \\
& =\int_{\Omega_{z}}(-\mathrm{i} 2 \pi \boldsymbol{\xi}) \mathrm{e}^{-\mathrm{i} 2 \pi \boldsymbol{z} \cdot \boldsymbol{\xi}} \chi_{\Omega_{z}}(\boldsymbol{\xi}) \hat{T}(\boldsymbol{\xi}) \mathrm{d} \boldsymbol{\xi} .
\end{aligned}
$$

We define the limited aperture directional derivative of $T$ in the direction of the unit vector $\widehat{\boldsymbol{\mu}}$ as follows:

$$
\widehat{\boldsymbol{\mu}} \cdot \nabla_{\boldsymbol{z}} T(\boldsymbol{z})=(-\mathrm{i} 2 \pi) \int_{\Omega_{z}} \mathrm{e}^{-\mathrm{i} 2 \pi \boldsymbol{z} \cdot \boldsymbol{\xi}} \widehat{\boldsymbol{\mu}} \cdot \boldsymbol{\xi} \chi_{\Omega_{z}}(\boldsymbol{\xi}) \hat{T}(\boldsymbol{\xi}) \mathrm{d} \boldsymbol{\xi} .
$$

Next, we define the following operator:

$$
\Delta_{\widehat{\boldsymbol{\mu}}}^{p} T(\boldsymbol{z}):=\int_{\Omega_{z}} \mathrm{e}^{-\mathrm{i} 2 \pi \boldsymbol{z} \cdot \boldsymbol{\xi}}|\widehat{\boldsymbol{\mu}} \cdot \boldsymbol{\xi}|^{p} \chi_{\Omega_{z}}(\boldsymbol{\xi}) \hat{T}(\boldsymbol{\xi}) \mathrm{d} \boldsymbol{\xi}, \quad p \in \mathbb{R}
$$

The operator $\Delta_{\widehat{\mu}}^{p}$ can be interpreted as the $p^{t h}$ directional derivative for $p>0$, and $p^{t h}$ integral for $p<0$. Note that with this operator, magnitude of the gradient can be represented as

$$
\left|\nabla_{\boldsymbol{z}} T(\boldsymbol{z})\right|^{2}=\Delta_{\widehat{e}_{1}}^{2} T(\boldsymbol{z})+\Delta_{\widehat{e}_{2}}^{2} T(\boldsymbol{z})
$$

where $\widehat{\boldsymbol{e}}_{i}, i=1,2$ is the $i^{\text {th }}$ column of the $2 \times 2$ identity matrix.

With the modified PSF, filter $\bar{Q}$ becomes

$$
\bar{Q}(\boldsymbol{\xi})=\alpha_{p}\left|\widehat{\boldsymbol{\mu}}_{p} \cdot \boldsymbol{\xi}\right|^{p} Q(\boldsymbol{\xi})+\alpha_{q}\left|\widehat{\boldsymbol{\mu}}_{q} \cdot \boldsymbol{\xi}\right|^{q} Q(\boldsymbol{\xi})
$$

where $p>0$ and $q<0, \alpha_{p}, \alpha_{q} \geq 0$ and $\widehat{\boldsymbol{\mu}}_{p}, \widehat{\boldsymbol{\mu}}_{q}$ are two possibly orthogonal unit directions. The directional derivative of the image is enhanced along $\widehat{\boldsymbol{\mu}}_{p}$ while it is smoothed in the direction of $\widehat{\boldsymbol{\mu}}_{q}$. Note that if $p=q=0$, then $\bar{Q}(\boldsymbol{\xi})=Q(\boldsymbol{\xi})$; and if $\widehat{\boldsymbol{\mu}}_{q}=\widehat{\boldsymbol{\mu}}_{p}^{\perp}$, then the derivative of the image is enhanced in the direction of $\widehat{\boldsymbol{\mu}}_{p}$ while it is smoothed in the direction perpendicular to $\widehat{\boldsymbol{\mu}}_{p}$.

More generally, we define

$$
\bar{Q}(\boldsymbol{\xi})=\sum_{i} \alpha_{i}\left|\widehat{\boldsymbol{\mu}}_{p_{i}} \cdot \boldsymbol{\xi}\right|^{p_{i}} Q(\boldsymbol{\xi})
$$

where $\alpha_{i}>0$ and $p_{i} \in \mathbb{R}$ so that we can enhance (or smooth) edges in different directions. 


\subsection{Point Spread Function of the Edge Enhanced Reconstruction}

With new filter given in (19), the PSF of the edge enhancing (smoothing) reconstruction operator becomes

$$
L(\boldsymbol{z}, \boldsymbol{x}) \approx \sum_{i} \alpha_{p_{i}} \int \mathrm{e}^{2 \pi \mathrm{i} \boldsymbol{\xi} \cdot(\boldsymbol{z}-\boldsymbol{x})}\left|\widehat{\boldsymbol{\mu}}_{i} \cdot \boldsymbol{\xi}\right|^{p_{i}} \mathrm{~d} \boldsymbol{\xi} .
$$

Moreover, with this choice of PSF, the location and orientation of the edges are preserved.

- If $m=\arg \max p_{i}$, and $p_{m}>0$, the strength of the edges is increased (enhanced) by an order of $p_{m}$ in the $\{i\}$ direction of $\boldsymbol{\mu}_{m}$.

- If $n=\arg \min p_{i}$ and $p_{n}<0$, the strength of the edges is decreased (smoothed) by an order of $p_{n}$ in the $\{i\}$ direction of $\boldsymbol{\mu}_{n}$.

Thus, with this choice of filter we can control the directions along which we want to enhance (or suppress) the edges.

\section{NUMERICAL EXPERIMENTS}

In our numerical simulations, we considered two scenes. In the first scene, there is a square target of size $4.9 \mathrm{~km}$ and a rectangular target of size $4.9 \mathrm{~km}$ by $9.8 \mathrm{~km}$ located in a scene of size $[0,22] \times[0,22] \mathrm{km}^{2}$ with their centers located at $(-4.1,-0.7) \mathrm{km}$ and $(4.5,-4.1) \mathrm{km}$. In the second scene, we have a square target of size $12.9 \mathrm{~km}$ by $12.9 \mathrm{~km}$ with its center located at $(2.2,-0.4) \mathrm{km}$, and there is a 1 pixel-wide $(86 \mathrm{~m})$ wire-like line that makes a $26^{\circ}$ angle with the horizontal axis inside the square.

We discretize the scene with $256 \times 256$ pixels, where $(-11,-11,0) \mathrm{km},(0,0,0) \mathrm{km}$ and $(11,11,0) \mathrm{km}$ corresponds to the pixels $(1,1),(128,128)$ and $(256,256)$, respectively.

We take $A_{T R}(\boldsymbol{x}, \omega, s)=1$, which corresponds to an isotropic antenna radiating a delta-like impulse and compensation of geometric spreading factors in the data. Then, using the definition of Dirac-delta function and its homogeneity property, we have

$$
d(s, t) \approx c_{0} \int \boldsymbol{\delta}\left(c_{0} t-2|\mathbf{x}-\gamma(s)|\right) T(\boldsymbol{x}) \mathrm{d} \boldsymbol{x} .
$$

We used a discrete version of (21) to generate our simulation data. In these experiments, we considered a circular flight trajectory $\gamma(s)=(22 \cos s, 22 \sin s, 6.5) \mathrm{km}$ uniformly sampled for $s \in[0,2 \pi]$ at 512 points. (See Figure 1)

Figures 2,3,4,5,6 show reconstructed (part a) and thresholded (part b) images with different values for $p, \alpha$ and $\boldsymbol{\mu} . p$ denotes the order of derivative (or anti-derivative) operation, $\alpha$ controls the strength of the edges in the final image and $\boldsymbol{\mu}$ is the direction along which we want to enhance (or smooth) edges.

To threshold the final reconstructed image an iterative thresholding method given in Algorithm 1 is used .

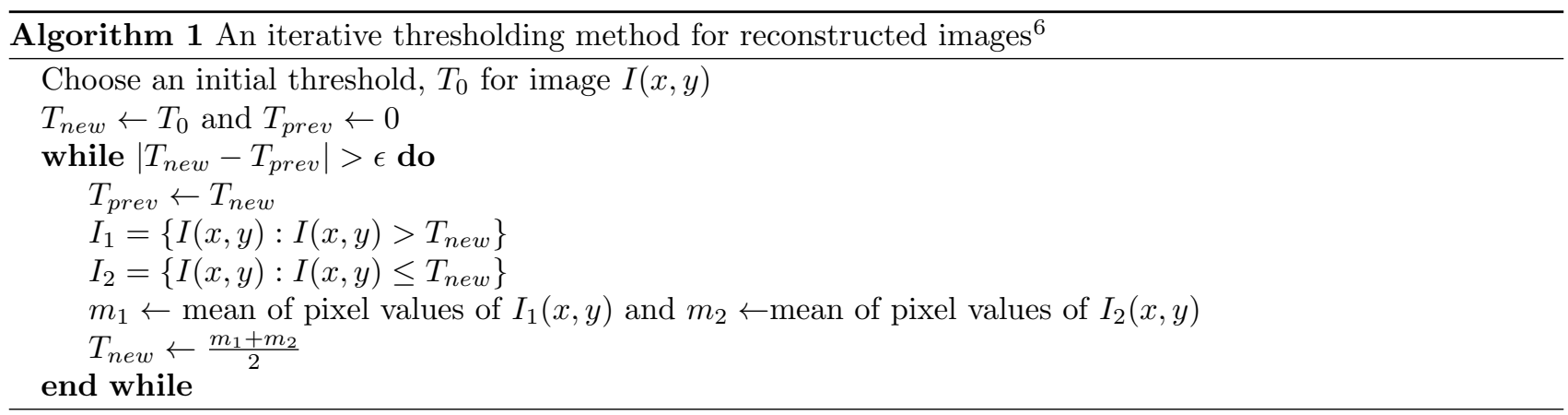


In Figures 2 and 3, edges are enhanced in all directions. In Figure 4, edges along y-direction are enhanced with $\boldsymbol{\mu}=(0,1)$. In Figure 5, edges along x-direction are enhanced with $\boldsymbol{\mu}=(1,0)$. Finally in Figure 6 , edges along $\mathrm{x}$ and y-directions are enhanced with $\boldsymbol{\mu}_{1}=(0,1)$ and $\boldsymbol{\mu}_{2}=(1,0)$. Note that, in Figures $4,5,6$ wire is not reconstructed since its direction is not along $\boldsymbol{\mu}$ 's used in the examples.

The numerical simulations show that the edges are enhanced (or smoothed) in desired directions and the edges are reconstructed directly from SAR data successfully.

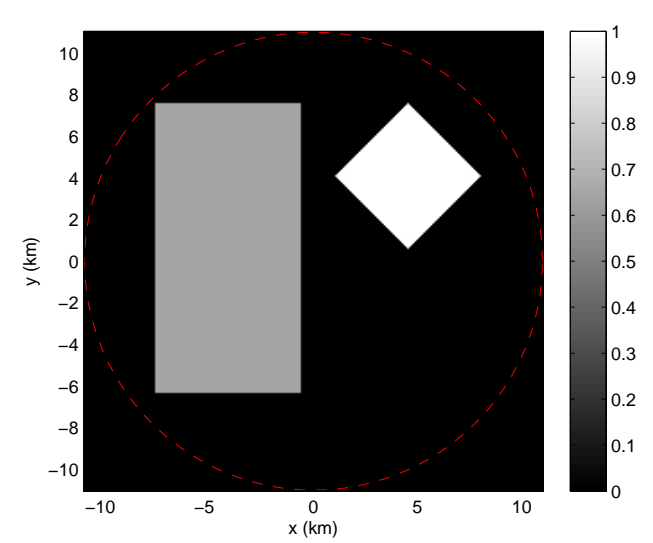

(a) Scene 1

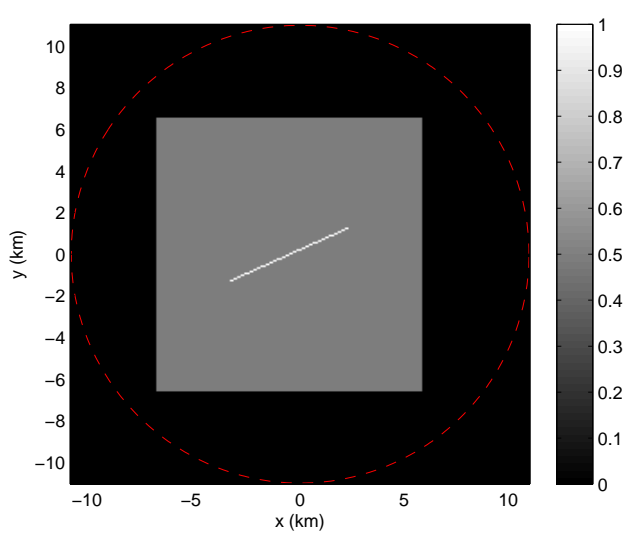

(b) Scene 2

Figure 1. Simulation setups

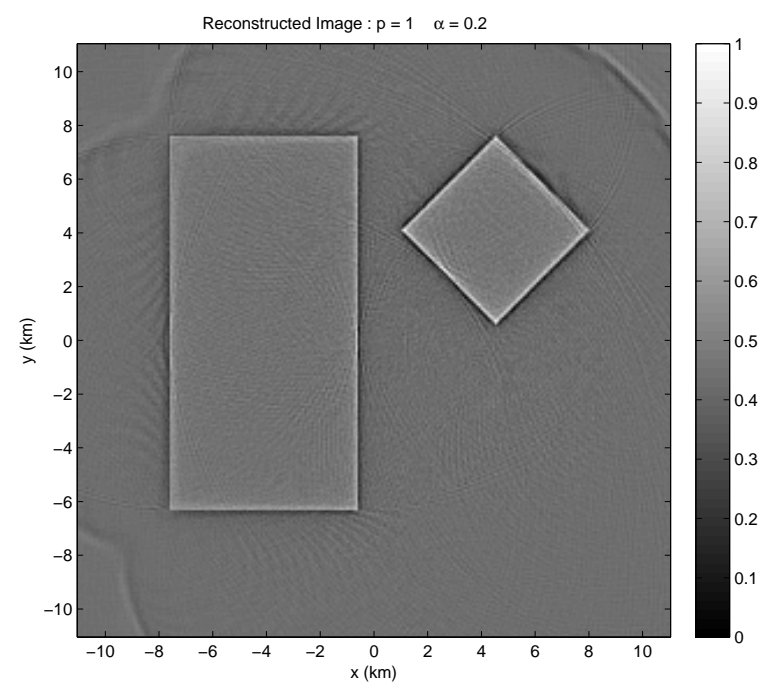

(a) Reconstructed image

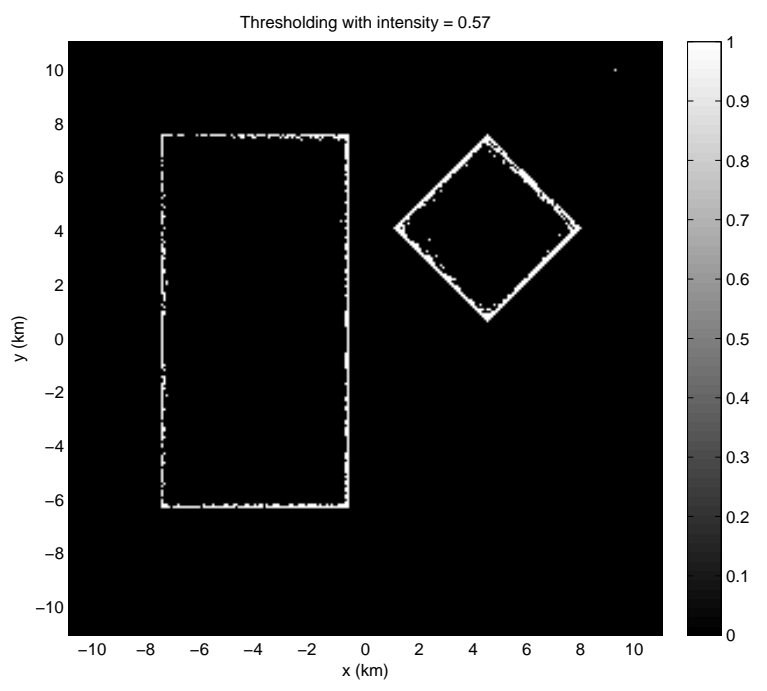

(b) Thresholded image

Figure 2. Enhancement of edges in all directions for scene 1. 


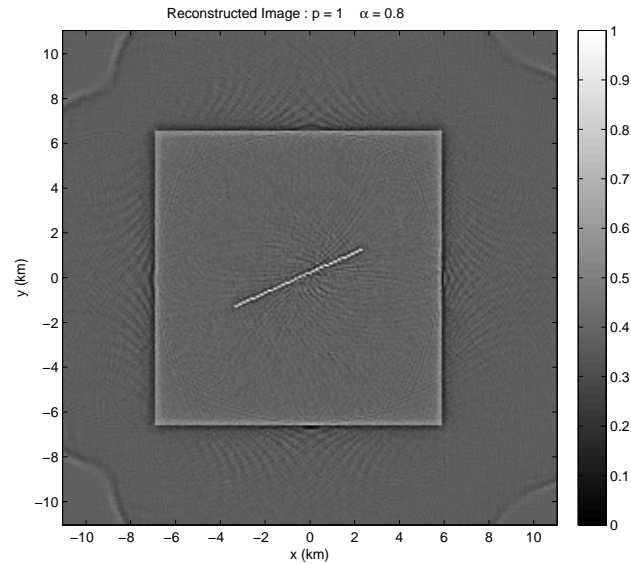

(a) Reconstructed image

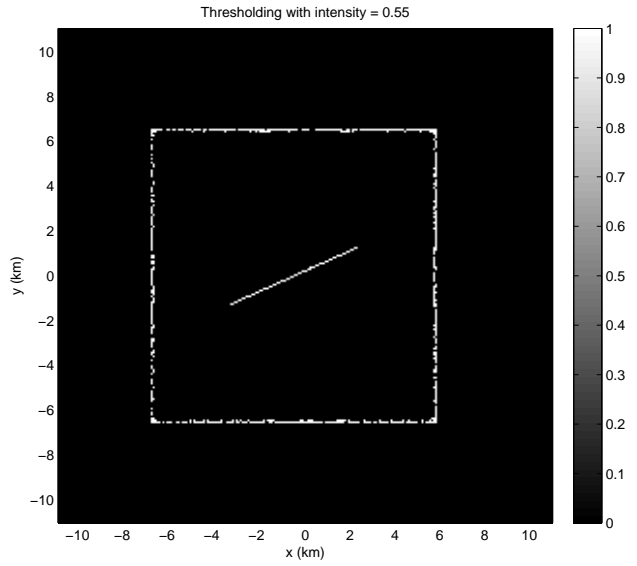

(b) Thresholded image

Figure 3. Enhancement of edges in all directions for scene 2.

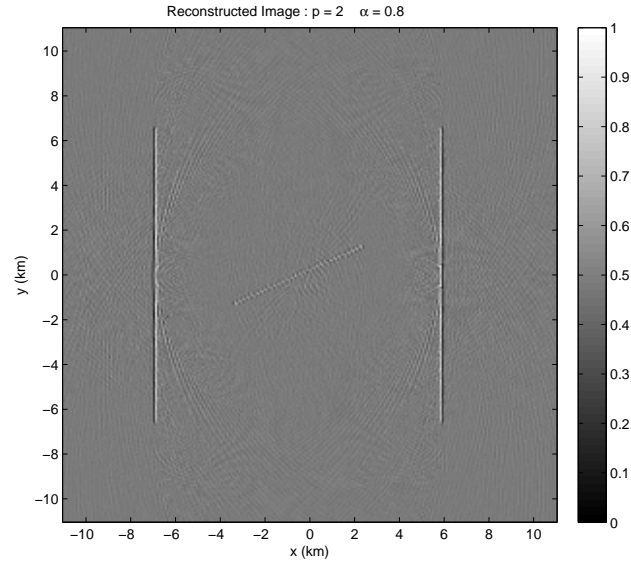

(a) Reconstructed image

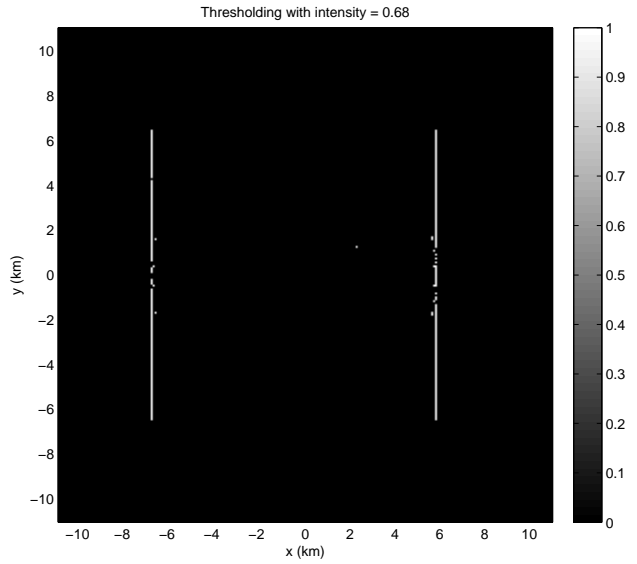

(b) Thresholded image

Figure 4. Enhancement of edges in $x$-direction for scene 2 with $\boldsymbol{\mu}=(1,0)$.

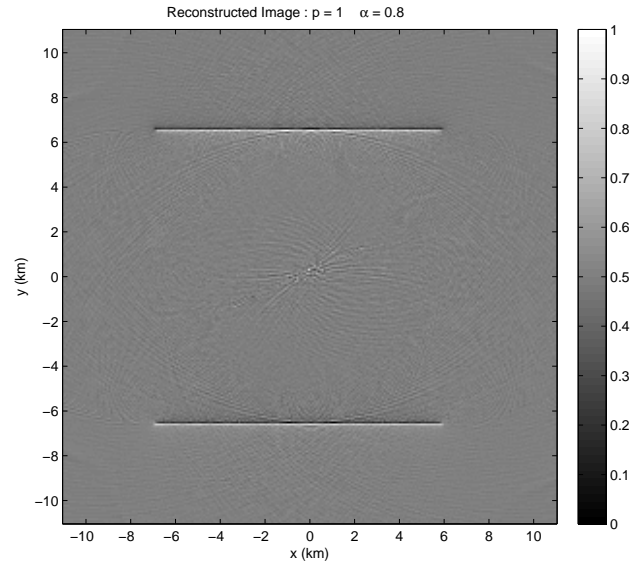

(a) Reconstructed image

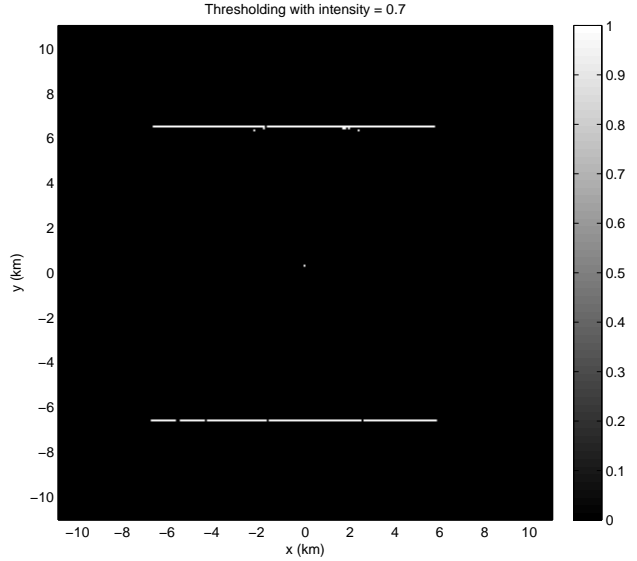

(b) Thresholded image

Figure 5. Enhancement of edges in $y$-direction for scene 2 with $\boldsymbol{\mu}=(0,1)$. 


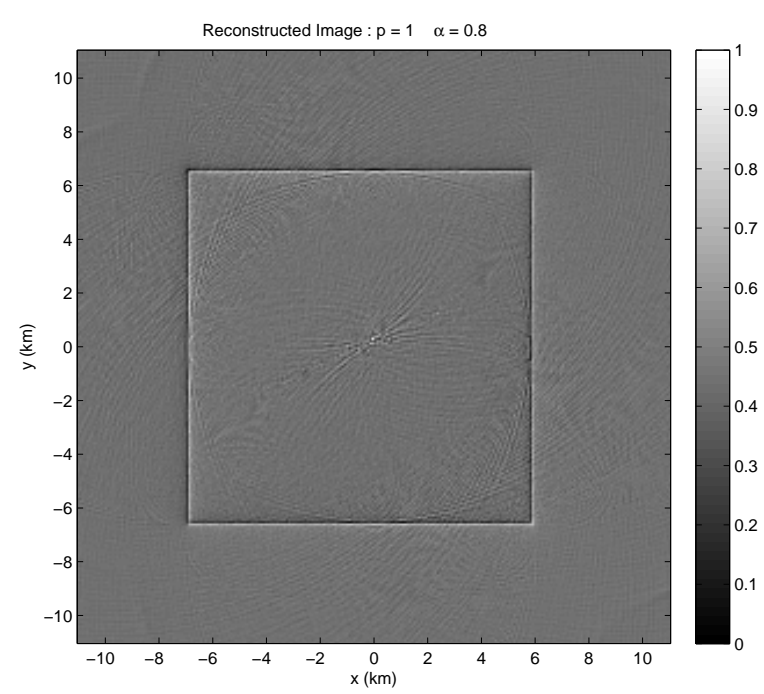

(a) Reconstructed image

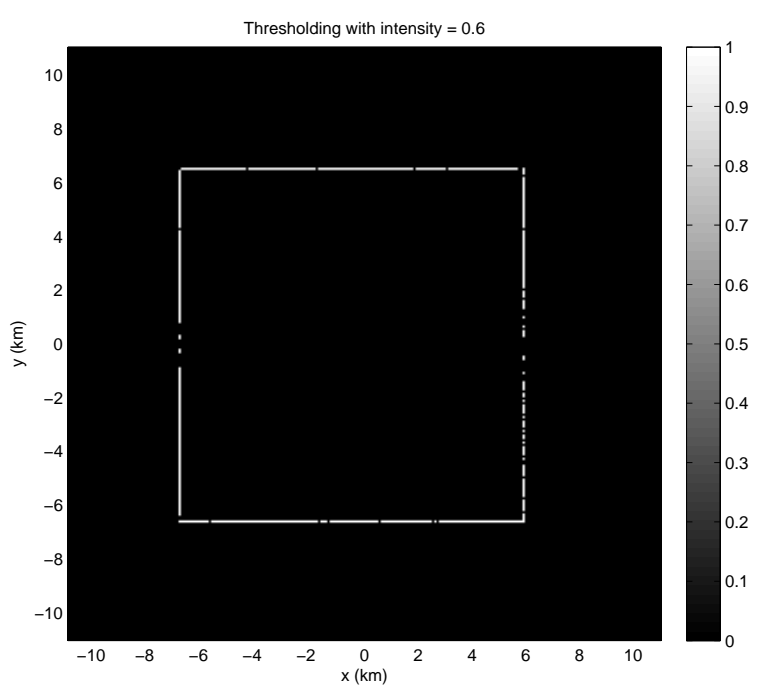

(b) Thresholded image

Figure 6 . Enhancement of edges in $x$ and $y$-directions for scene 2 with $\boldsymbol{\mu}_{1}=(1,0)$ and $\boldsymbol{\mu}_{2}=(0,1)$.

\section{CONCLUSION}

We presented a novel edge detection method applicable directly to SAR received signal. Our method first filters the received data, and then backprojects. The filter detects the edges of the scene in different directions at each pixel reconstructed. The method is computationally efficient and may be implemented with the computational complexity of the fast-backprojection algorithms. In our future work, we will discuss edge enhancement and detection in the presence of measurement noise.

\section{Acknowledgment}

This work was supported by the Air Force Office of Scientific Research (AFOSR) under the agreements FA955007-1-0363 and FA9550-09-1-0013, by the National Science Foundation (NSF) under Grant No. CCF-08030672, and the U.S. Department of Homeland Security under Award Number 2008-ST-061-ED0001.

\section{REFERENCES}

[1] Yarman, C., Yazıcı, B., and Cheney, M., "Bistatic synthetic aperture radar imaging for arbitrary flight trajectories," IEEE Transactions on Image Processing 17, 84-93 (2008).

[2] Nolan, C. J. and Cheney, M., "Synthetic aperture inversion," Inverse Problems 18, 221-236 (2002).

[3] Duistermaat, J. J., [Fourier Integral Operators], Birkhauser, Boston (1996).

[4] Treves, F., [Introduction to Pseudodifferential and Fourier Integral Operators, volumes I and II], Plenum Press, New York (1980).

[5] Grigis, A. and Sjöstrand, J., [Microlocal Analysis for Differential Operators: An Introduction], London Mathematical Society Lecture Note Series, Vol. 196, Cambridge University Press, Cambridge (1994).

[6] González, R. and Woods, R., [Digital Image Processing], Prentice-Hall, Inc., New Jersey, 3rd ed. (2008).

[7] Canny, J., "A computational approach to edge detection," Readings in computer vision: issues, problems, principles, and paradigms 184, 87-116 (1987). 